\title{
CONTROLE BIOLÓGICO
}

\section{Efeito do Parasitismo por Glyptapanteles muesebecki (Blanchard) no Consumo e Utilização do Alimento por Pseudaletia sequax Franclemont}

\author{
Augusta K. Doetzer ${ }^{1}$ e Luís A. Foerster ${ }^{1}$ \\ ${ }^{1}$ Departamento de Zoologia, UFPR, Caixa postal 19020, 81531-990, Curitiba, PR.
}

An. Soc. Entomol. Brasil 27(2): 255-264 (1998)

Effect of Parasitism by Glyptapanteles muesebecki (Blanchard) on Food Consumption and Utilization by Pseudaletia sequax Franclemont

\begin{abstract}
Food consumption and utilization for unparasitized larvae of Pseudaletia sequax Franclemont (Lepidoptera: Noctuidae) in relation to parasitized larvae with Glyptapanteles muesebecki (Blanchard) (Hymenoptera: Braconidae) was evaluated in laboratory, using kikuyo grass (Pennisetum clandestinum) as larval food. Parasitized larvae consumed significantly less foliage than unparasitized ones, although the larval stage of parasitized larvae was longer than the healthy ones. Parasitized larvae which had seven instars produced more parasitoids and consumed significantly more foliage than parasitized larvae which had six instars. Relative consumption rate (RCR), relative metabolic rate (RMR) and approximate digestibility (AD) were significantly higher in parasitized larvae, but the relative growth rate (RGR) and the efficiency of ingested (ECI) and digested (ECD) food were higher in unparasitized larvae. $G$. muesebecki regulates growth and development of $P$. sequax through the number of eggs laid. The values obtained for the number of pupae/host were positively correlated with food consumption and final weight of the host. The development time of the parasitoid was also positively correlated to the number of pupae/host.
\end{abstract}

KEY WORDS: Insecta, biological control, parasitoid, host regulation, armyworm.

RESUMO - O consumo e a utilização do alimento em lagartas de Pseudaletia sequax Franclemont sadias e parasitadas por Glyptapanteles muesebecki (Blanchard) (Hymenoptera: Braconidae) foram avaliados em laboratório, utilizando-se folhas de capim quicuio (Pennisetum clandestinum). Lagartas parasitadas consumiram significativamente menos alimento que lagartas não parasitadas, embora o parasitismo tenha provocado um aumento na duração total do período de alimentação. Lagartas parasitadas que passaram por sete ínstares, pelo fato de terem dado origem a um número maior de parasitóides, consumiram significativamente mais alimento que lagartas com seis ínstares. Os valores encontrados para a taxa de consumo relativo (RCR), taxa metabólica relativa (RMR) e digestibilidade aproximada (AD) foram superiores em lagartas parasitadas. Por outro lado, a taxa de crescimento relativo (RGR) e os valores 
de eficiência de conversão do alimento ingerido (ECI) e digerido (ECD) em biomassa foram mais altos em lagartas não parasitadas. G. muesebecki regulou o crescimento e o desenvolvimento de $P$. sequax através do número de ovos depositados, visto que o número de pupas do parasitóide por lagarta foi positivamente correlacionado com o consumo total do alimento e o peso final do hospedeiro. O tempo de desenvolvimento ovo-larval do parasitóide também foi positivamente correlacionado com o número de parasitóides por lagarta.

PALAVRAS-CHAVE: Insecta, controle biológico, parasitóide, regulação do hospedeiro, lagarta do trigo.

Insetos parasitóides podem causar inúmeros efeitos na fisiologia de seus hospedeiros. O conhecimento destes efeitos é de grande importância, visto que o sucesso de um parasitóide depende, em geral, da sua habilidade em regular a fisiologia do inseto parasitado (Vinson \& Iwantsch 1980). Tal regulação tem, freqüentemente, forte influência sobre o consumo alimentar do hospedeiro (Senthamizhselvan \& Muthukrishnan 1989). De acordo com Hill (1986), parasitóides que causam uma redução significativa no consumo do alimento pelo hospedeiro são considerados eficientes como agentes de controle biológico por reduzirem o prejuízo causado pela praga. Segundo Smith \& Smilowitz (1976), a regulação no desenvolvimento do hospedeiro pelo parasitóide pode ser diferente em parasitóides gregários e solitários, já que espécies gregárias requerem uma maior quantidade de alimento. Desta forma, estes autores concluíram que parasitóides gregários tendem a aumentar o consumo alimentar por parte de seus hospedeiros, enquanto que, em geral, parasitóides solitários reduzem a alimentação dos hospedeiros. Em vista disto, Slansky Jr. (1978) propôs que parasitóides solitários são mais úteis em programas de controle biológico.

Ao contrário do postulado por Smith \& Smilowitz (1976) e Slansky Jr. (1978), diversos autores constataram que espécies gregárias de braconídeos também causam uma redução significativa no consumo alimentar do hospedeiro e podem ser considerados eficientes agentes de controle (Duodu \& Antoh 1984, Beckage \& Templeton 1986, Hill 1986, Tanaka et al. 1992). No entanto, os relatos existentes na literatura demonstram que o impacto de braconídeos gregários na redução dos danos causados pelas pragas é menor em relação a parasitóides solitários, variando de $28 \%$ a $69 \%$.

Em poucos relatos o consumo alimentar do hospedeiro aumentou devido ao parasitismo, sendo que todos estes estudos referem-se ao braconídeo gregário Cotesia glomerata (L.) (Rahman 1970, Smith \& Smilowitz 1976, Slansky Jr. 1978).

Muitos parasitóides regulam o crescimento e o desenvolvimento de seus hospedeiros de acordo com o número de ovos depositados (Hill 1986, Sato et al. 1986, Tanaka et al. 1992). O tempo necessário para o desenvolvimento do parasitóide também pode ser dependente do número de ovos depositados (Beckage \& Riddiford 1978, Hill 1986).

Este trabalho foi realizado com o objetivo de verificar o efeito do parasitismo pelo endoparasitóide gregário Glyptapanteles muesebecki (Blanchard) no consumo e utilização do alimento por Pseudaletia sequax Flanclemont, a fim de verificar o potencial deste parasitóide como agente natural de controle da lagarta do trigo.

\section{Material e Métodos}

$\mathrm{O}$ experimento foi realizado em câmara climatizada a $21^{\circ} \pm 1^{\circ} \mathrm{C}$, fotofase de 12 horas 
e U.R. $70 \pm 5 \%$. Quarenta fêmeas do parasitóide previamente acasaladas foram individualizadas em tubos de ensaio $(9 \mathrm{x}$ $3 \mathrm{~cm}$ ), recebendo uma lagarta de $2^{\circ}$ ínstar para o parasitismo. Após 24 horas, as lagartas foram transferidas para potes de polietileno de $7 \mathrm{~cm}$ de altura por $4 \mathrm{~cm}$ de diâmetro, sendo alimentadas com folhas de capim quicuio (Pennisetum clandestinum). Simultaneamente, 40 lagartas não parasitadas foram individualizadas a partir do $2^{\circ}$ ínstar e criadas de forma idêntica às parasitadas.

Para verificação do peso médio das lagartas no início do experimento, 60 lagartas de $2^{\circ}$ ínstar foram, em 3 grupos de 20 exemplares, mortas por congelamento, levadas à estufa entre $70^{\circ}$ e $80^{\circ} \mathrm{C}$ por 48 horas e pesadas a seguir. As avaliações dos demais parâmetros foram iniciadas a partir do $3^{\circ}$ ínstar. Diariamente, foi pesado a fresco o alimento ofertado às lagartas e, após 24 horas, o alimento não consumido e as fezes produzidas foram levados à estufa e pesados. O peso seco do alimento ofertado foi estimado através da multiplicação do peso fresco das folhas fornecidas pela percentagem média do peso seco de uma folha, obtida através da média do peso seco de 20 folhas. Para as lagartas sadias, este procedimento foi mantido até que as lagartas atingissem a fase pré-pupal, quando foram mortas por congelamento, levadas à estufa e pesadas. Para as lagartas parasitadas, seguiu-se este procedimento até a emergência dos parasitóides, quando as lagartas mortas foram levadas à estufa e pesadas a seguir. Registrou-se ainda a duração de cada ínstar e de todo o período de alimentação de $P$. sequax.

Os índices nutricionais, conforme proposto por Waldbauer (1968), com as modificações feitas por Scriber \& Slansky Jr. (1981), foram determinados para lagartas sadias e parasitadas.

A análise dos resultados foi realizada separadamente para lagartas que passaram por 6 e 7 ínstares durante seu desenvolvimento. Comparações estatísticas entre lagartas parasitadas e não parasitadas foram realizadas através do teste "t" ao nível de $5 \%$ de probabilidade. Através da análise de regressão, o número de pupas do parasitóide por hospedeiro foi relacionado com o consumo total do alimento e com o peso final de cada lagarta, bem como com o tempo necessário para o desenvolvimento do parasitóide.

\section{Resultados e Discussão}

O parasitismo por G. muesebecki reduziu significativamente o consumo de alimento em lagartas de P. sequax (Tabela 1 e Figs. 1, 2), como observado para outros lepidópteros parasitados por braconídeos gregários (Duodu \& Antoh 1984, Beckage \& Templeton 1986, Hill 1986, Tanaka 1992).

O tempo de alimentação de $P$. sequax foi aumentado devido ao parasitismo, sem contudo ocorrer aumento na quantidade de alimento ingerido. Para lagartas sadias com seis ínstares a duração média ( \pm D.P.) do período de alimentação foi de 27,1 $\pm 1,0$ dias, enquanto que para lagartas parasitadas foi de $30,7 \pm 2,4$. Lagartas sadias com sete ínstares alimentaram-se por $36,1 \pm 1,9$ dias, enquanto que lagartas parasitadas alimentaram-se por $37,8 \pm 2,1$. Estes resultados discordam daqueles obtidos por Duodu \& Antoh (1984) para lagartas de Sylepta derogata (F.) sadias e parasitadas por Apanteles sagax Wilkinson, visto que os autores citam que um período mais curto de alimentação observado em lagartas parasitadas pode ser uma das causas da redução no consumo alimentar desta espécie após o parasitismo.

O número de pupas do parasitóide por lagarta foi positivamente correlacionado com o consumo total do alimento e com o peso final do hospedeiro (Figs. 3, 4). Para outros braconídeos, a correlação positiva entre o número de parasitóides e o peso final do hospedeiro também foi constatada (Sato et al. 1986, Tanaka et al. 1992). Hill (1986) encontrou correlação positiva entre o consumo alimentar de $P$. separata Walker e o número de adultos de Cotesia ruficrus Haliday obtido por hospedeiro. O número de pupas de G. muesebecki obtidas por 
Tabela 1. Média ( \pm D.P) de consumo alimentar, produção de fezes, ganho de peso, peso médio, alimento assimilado, alimento metabilozado, número de parasitóides por hospedeiro e desenvolvimento ovo-larval do parasitóide em lagartas de Pseudaletia sequax sadias e parasitadas por Glyptapanteles muesebecki.

\begin{tabular}{|c|c|c|c|c|}
\hline \multirow[b]{2}{*}{$\begin{array}{l}\text { Parâmetro avaliado }{ }^{1} \\
\text { (mg peso seco) }\end{array}$} & \multicolumn{2}{|c|}{ Lagartas com seis ínstares } & \multicolumn{2}{|c|}{ Lagartas com sete ínstares } \\
\hline & $\begin{array}{l}\text { Sadias } \\
(n=21)\end{array}$ & $\begin{array}{c}\text { Parasitadas } \\
(\mathrm{n}=23)\end{array}$ & $\begin{array}{l}\text { Sadias } \\
(\mathrm{n}=18)\end{array}$ & $\begin{array}{l}\text { Parasitadas } \\
(\mathrm{n}=15)\end{array}$ \\
\hline Consumo alimentar & $791,9 \pm 64,4 \mathrm{a}$ & $346,5 \pm 127,1 b$ & $802,6 \pm 84,1 \mathrm{a}$ & $593,3 \pm 98,3 b$ \\
\hline Produção de fezes & $444,7 \pm 43,3 \mathrm{a}$ & $185,7 \pm 80,5 b$ & $425,0 \pm 35,5 a$ & $295,756,2 b$ \\
\hline Ganho de peso & $108,1 \pm 8,3 \mathrm{a}$ & $19,5 \pm 6,4 b$ & $109,2 \pm 15,8 \mathrm{a}$ & $26,5 \pm 3,5 b$ \\
\hline Peso médio & $54,0 \pm 4,2 \mathrm{a}$ & $9,7 \pm 3,2 b$ & $54,6 \pm 7,9 \mathrm{a}$ & $13,2 \pm 1,8 b$ \\
\hline Alimento assimilado & $347,2 \pm 22,8 \mathrm{a}$ & $160,8 \pm 51,3 b$ & $377,5 \pm 54,2 \mathrm{a}$ & $297,9 \pm 50,0 \mathrm{~b}$ \\
\hline Alimento metabolizado & $239,1 \pm 21,4 \mathrm{a}$ & $141,3 \pm 47,9 b$ & $268,3 \pm 46,5 a$ & $271,1 \pm 47,3 \mathrm{a}$ \\
\hline Número de parasitóides por hospedeiro & - & $61,8 \pm 28,3$ & - & $115,7 \pm 36,9$ \\
\hline Desenvolvimento ovo-larval do parasitóide (dia & ias) - & $23,7 \pm 2,4$ & - & $30,7 \pm 1,4$ \\
\hline
\end{tabular}

${ }^{1}$ Médias seguidas de mesma letra, no sentido horizontal, não diferem estatisticamente pelo teste " $\mathrm{t}$ " ao nível de 5\% de probabilidade. Análises realizadas separadamente para lagartas com seis e sete ínstares.

hospedeiro foi também positivamente correlacionado com a duração do período ovo-larval do parasitóide, quando lagartas com seis e sete ínstares foram analisadas conjuntamente (Fig. 5). No entanto, quando as análises foram realizadas separadamente

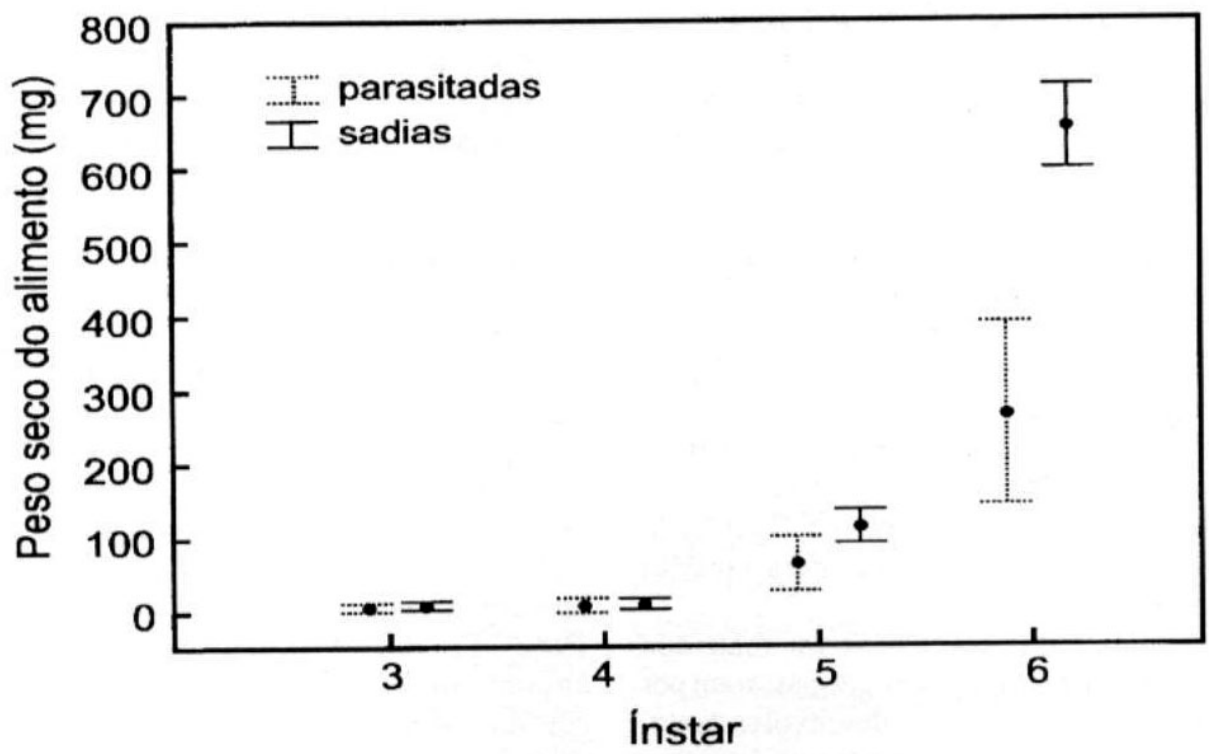

Figura 1. Consumo médio por ínstar em lagartas de Pseudaletia sequax, com seis ínstares, sadias e parasitadas por Glyptapanteles muesebeck. 


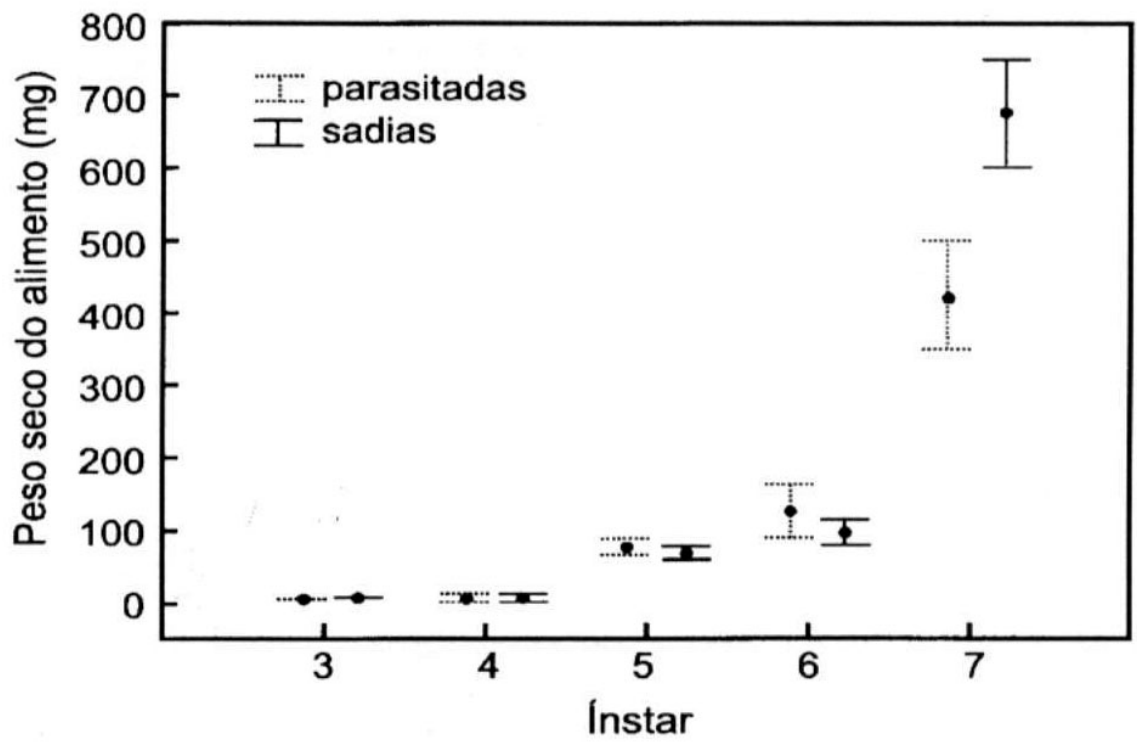

Figura 2. Consumo médio por ínstar em lagartas de Pseudaletia sequax, com sete ínstares, sadias e parasitadas por Glyptapanteles muesebecki.

para lagartas com seis e sete ínstares, esta correlação foi estatisticamente positiva somente para lagartas com sete ínstares.
Resultados semelhantes foram encontrados por Beckage \& Riddiford (1978) e Hill (1986), visto que estes autores verificaram que

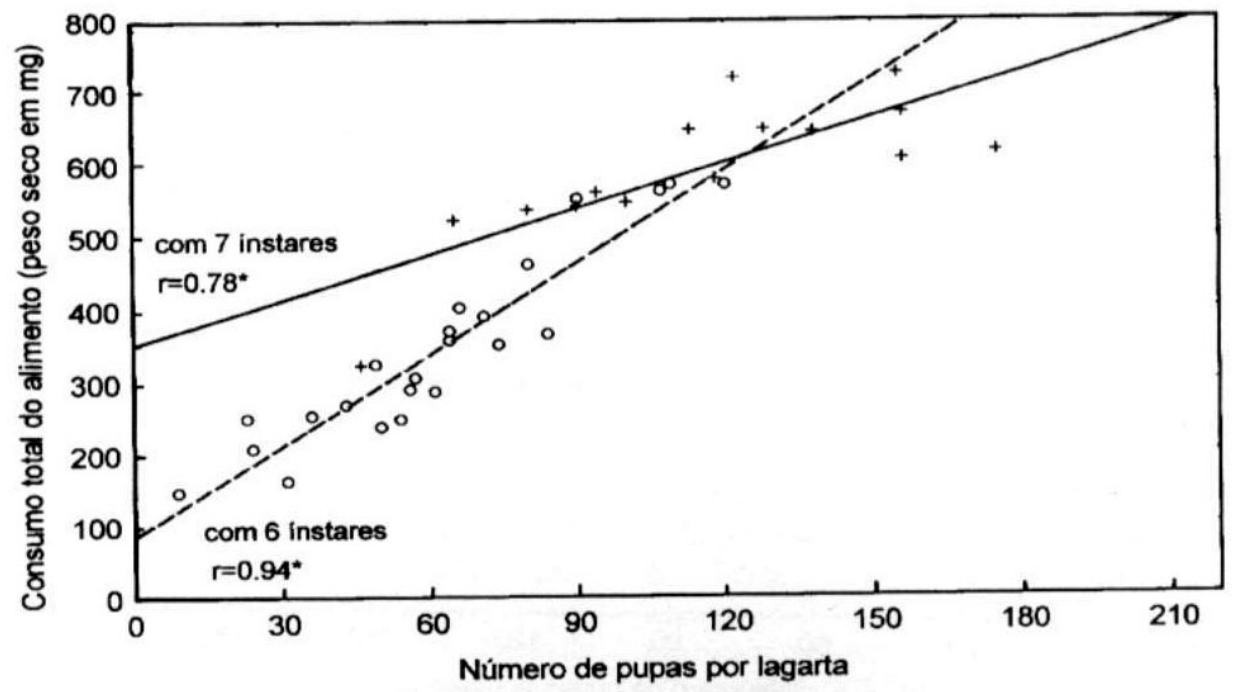

Figura 3. Correlação entre o número de pupas de Glyptapanteles muesebecki obtidas por lagarta de Pseudaletia sequax, com seis e sete ínstares, e o consumo total do alimento pelo hospedeiro. *Correlações são significativas ao nível de $5 \%$ de probabilidade. 


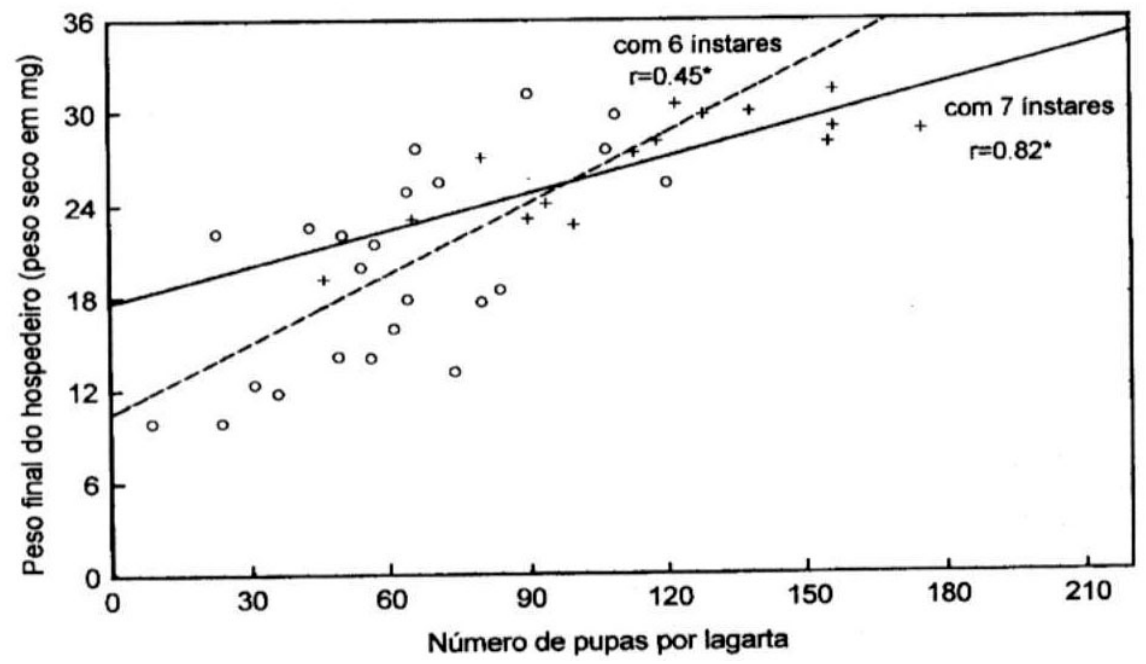

Figura 4. Correlação entre o número de pupas de Glyptapanteles muesebecki obtidas por lagarta de Pseudaletia sequax, com seis e sete ínstares, e o peso final do hospedeiro. *Correlações são significativas ao nível de 5\% de probabilidade.

o tempo de desenvolvimento de Apanteles congregatus Say e C.ruficrus foi dependente do número de parasitóides obtidos por lagarta.
Lagartas com sete ínstares parasitadas consumiram mais alimento que lagartas com seis ínstares, sendo que isto é explicado pelo fato de um número maior de parasitóides por

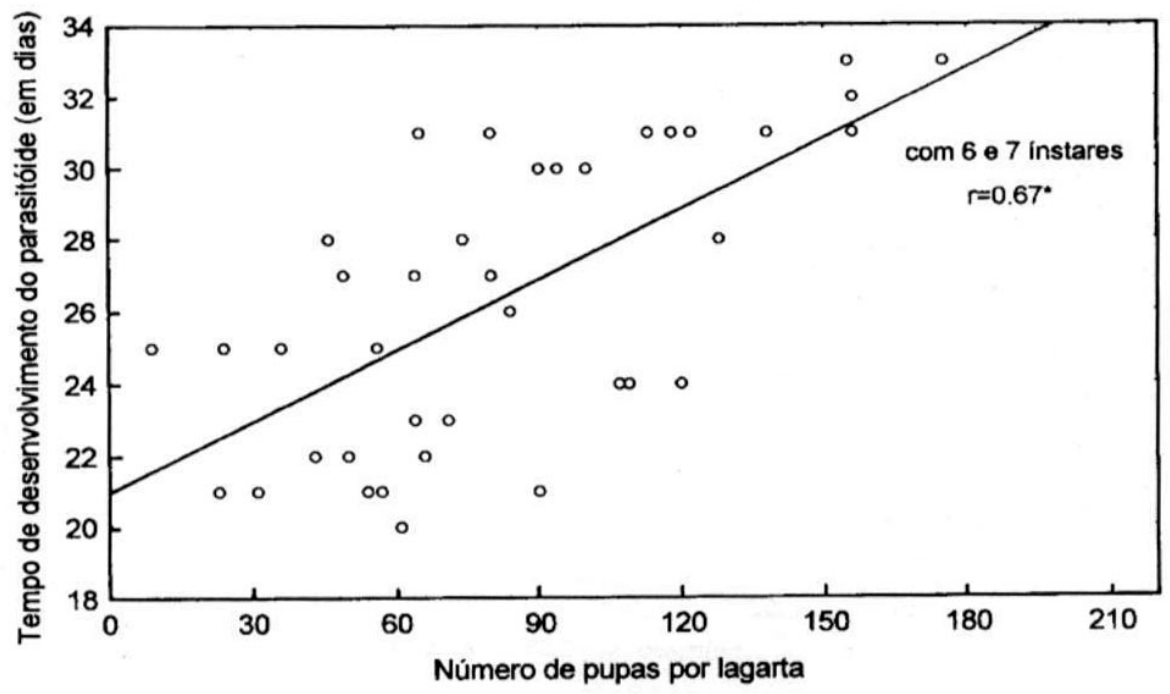

Figura 5. Correlação entre o número de pupas de Glyptapanteles muesebecki obtidas por lagarta de Pseudaletia sequax, com seis e sete ínstares, e o tempo de desenvolvimento do parasitóide. *Correlações são significativas ao nível de 5\% de probabilidade. 
hospedeiro ter sido produzido em lagartas com sete ínstares (Tabela 1). Resultados semelhantes foram obtidos por Hill (1986) para $C$. ruficrus parasitando P. separata. Em vista disto, em relação às lagartas com seis ínstares, que consumiram cerca de 56\% menos alimento que lagartas sadias, a redução no consumo alimentar devido ao parasitismo foi nitidamente menor para lagartas com sete ínstares (26\%). A quantidade de alimento consumido por lagartas de $P$. sequax sadias e parasitadas por $G$. muesebecki foi comparada por Oliveira $\mathrm{F}^{\circ}(1984)$ através da pesagem das fezes e os resultados descritos foram semelhantes aos encontrados neste trabalho. O autor encontrou uma redução de 46,2\% na produção de fezes de $P$. sequax após o parasitismo, sem contudo especificar o número de ínstares dos exemplares estudados.

A análise conjunta de lagartas com seis e sete ínstares demonstra que o impacto do parasitismo por G. muesebecki no consumo alimentar de $P$. sequax, em média de $41 \%$, supera A. flavipes Cameron (Senthamizhselvan \& Muthukrishnan 1989) e A. sagax Wilkinson (Duodu \& Antoh 1984), outros braconídeos gregários que reduzem o consumo do hospedeiro em $28 \%$ e $30 \%$, respectivamente. De acordo com Hill (1986), a percentagem de redução no consumo alimentar de $P$. separata devido ao parasitismo por C. ruficrus é $49 \%$ para lagartas com sete ínstares e $72 \%$ para lagartas com seis ínstares, com uma redução estimada no campo de $69 \%$.

Observações pessoais em lagartas de $P$. sequax coletadas em pastagens de aveia e azevém entre 1993 e 1995 demonstraram que o número de parasitóides produzidos por hospedeiro na natureza é acentuadamente inferior aos valores encontrados neste trabalho. Lazzari \& Lazzari (1985) encontraram, em coletas de campo, uma média de 6,6 pupas de G. muesebecki por lagarta. Esta diferença indica a ocorrência de superparasitismo nos experimentos de laboratório, devido ao fato das fêmeas do parasitóide terem estado em contato com apenas uma lagarta hospedeira durante 24 horas. Com base na correlação existente entre o número de parasitóides por lagarta e o consumo alimentar do hospedeiro pode-se afirmar que, a nível de campo, a percentagem de redução nos danos causados por $P$. sequax após o parasitismo por $G$. muesebecki é superior a $26 \%$ e $56 \%$, valores encontrados neste trabalho para lagartas com sete e seis ínstares, respectivamente. Estes resultados indicam o potencial de G. muesebecki como agente natural de controle da lagarta do trigo.

O parasitismo por G. muesebecki influenciou significativamente a utilização do alimento consumido por P. sequax (Tabela 2). A RCR foi significativamente maior em lagartas parasitadas, apesar destas consumirem menor quantidade total de alimento, demonstrando que a quantidade de alimento consumido em relação ao peso corpóreo aumenta devido ao parasitismo. Ao contrário, os valores encontrados para a RGR foram superiores para lagartas sadias, visto que o parasitismo provocou um acréscimo na duração do período de alimentação de $P$. sequax. A RMR foi maior em lagartas parasitadas, demonstrando que estas utilizaram maior parte do alimento como energia metabólica. Senthamizhselvan \& Muthukrishnan (1989) também observaram que o parasitismo por $A$. flavipes e $A$. prodeniae aumentou a RCR e a RMR em lagartas de $P$. scintillans e $S$. exigua. Os valores obtidos de AD foram superiores para lagartas parasitadas, indicando que uma maior quantidade do alimento ingerido foi efetivamente assimilada por estes indivíduos. $\mathrm{O}$ aumento na $\mathrm{AD}$ devido ao parasitismo também foi observado para A. sagax parasitando $S$. derogata (Duodu \& Antoh 1984) e A. flavipes e A. prodeniae parasitando $P$. scintillans e $S$. exigua (Senthamizhselvan \& Muthukrishnan 1989). No entanto, Guillot \& Vinson (1973) e Jalali et al. (1988) observaram que o parasitismo por $C$. nigriceps e $C$. marginiventris reduz a $\mathrm{AD}$ em $H$. virescens e $S$. litura. Os valores de ECI foram significativamente maiores em lagartas sadias, demonstrando que estas possuem maior eficiência na conversão do alimento 
ingerido em biomassa. A ECD foi igualmente superior em lagartas sadias, evidenciando um melhor aproveitamento da porção digerível do alimento para conversão em substância corpórea. Guillot \& Vinson (1973) também constataram que o parasitismo por $C$. nigriceps reduz a ECI e a ECD em lagartas de $H$. virescens. Entretanto, Duodu \& Antoh (1984) verificaram que o parasitismo por $A$. sagax não afetou a ECI e a ECD em $S$. derogata. Para as relações entre $A$. glomeratus
ECI e ECD, mas somente sobre a AD, diferenciando de G. muesebecki, que provocou uma acentuada redução nos valores de ECI e ECD em $P$. sequax.

Comparando-se os índices nutricionais encontrados para lagartas sadias com seis e sete ínstares, verifica-se que, devido a um consumo alimentar e ganho de peso semelhantes entre estes índivíduos, com uma maior duração do período de alimentação em lagartas com sete ínstares, os valores de RCR,

Tabela 2. Média ( \pm D.P.) da taxa de consumo relativo (RCR), taxa de crescimento relativo (RGR), taxa metabólica relativa (RMR), digestibilidade aproximada (AD), eficiência de conversão do alimento ingerido (ECI) e digerido (ECD) e custo metabólico, em todo o período de alimentação, em lagartas de Pseudaletia sequax sadias e parasitadas por Glyptapanteles muesebecki.

\begin{tabular}{|c|c|c|c|c|}
\hline \multirow[b]{2}{*}{ Índices $^{1}$} & \multicolumn{2}{|c|}{ Lagartas com seis ínstares } & \multicolumn{2}{|c|}{ Lagartas com sete ínstares } \\
\hline & $\begin{array}{l}\text { Sadias } \\
(n=21)\end{array}$ & $\begin{array}{c}\text { Parasitadas } \\
\quad(\mathrm{n}=23)\end{array}$ & $\begin{array}{l}\text { Sadias } \\
(\mathrm{n}=18)\end{array}$ & $\begin{array}{c}\text { Parasitadas } \\
(\mathrm{n}=15)\end{array}$ \\
\hline $\mathrm{RCR}(\mathrm{mg} / \mathrm{mg} / \mathrm{dia})$ & $0,83 \pm 0,11 \mathrm{a}$ & $1,70 \pm 0,33 b$ & $0,55 \pm 0,07 \mathrm{a}$ & $1,57 \pm 0,15 b$ \\
\hline RGR (mg/mg/dia) & $0,11 \pm 0,01 \mathrm{a}$ & $0,10 \pm 0,01 b$ & $0,07 \pm 0,01 \mathrm{a}$ & $0,07 \pm 0,01 b$ \\
\hline RMR (mg/mg/dia) & $0,25 \pm 0,04 \mathrm{a}$ & $0,71 \pm 0,20 \mathrm{~b}$ & $0,18 \pm 0,04 a$ & $0,72 \pm 0,09 b$ \\
\hline $\mathrm{AD}(\%)$ & $43,9 \pm 1,2 \mathrm{a}$ & $47,5 \pm 5,7 b$ & $46,9 \pm 2,5 \mathrm{a}$ & $50,2 \pm 3,4 \mathrm{~b}$ \\
\hline $\mathrm{ECI}(\%)$ & $13,7 \pm 1,4 \mathrm{a}$ & $5,9 \pm 1,6 b$ & $13,6 \pm 1,6 a$ & $4,5 \pm 0,5 b$ \\
\hline $\operatorname{ECD}(\%)$ & $31,2 \pm 2,6 \mathrm{a}$ & $12,6 \pm 3,9 b$ & $29,1 \pm 3,7 \mathrm{a}$ & $9,0 \pm 1,1 b$ \\
\hline Custo metabólico (\%) & $68,8 \pm 2,6 a$ & $87,4 \pm 3,9 b$ & $70,9 \pm 3,7 \mathrm{a}$ & $91,0 \pm 1,1 b$ \\
\hline
\end{tabular}

${ }^{1}$ Médias seguidas de mesma letra, no sentido horizontal, não diferem estatisticamente pelo teste "t" ao nível de 5\% de probabilidade. Análises realizadas separadamente para lagartas com seis e sete ínstares.

e $P$. rapae, onde o parasitismo provocou um aumento no consumo alimentar, Slansky Jr. (1978) constatou que a ECD foi significativamente superior em lagartas parasitadas.

Os índices nutricionais encontrados por Duodu \& Antoh (1984) para lagartas não parasitadas de $S$. derogata assemelham-se aos obtidos para $P$. sequax. Os valores médios de $\mathrm{AD}, \mathrm{ECI}$ e ECD encontrados pelos autores foram $39,7 \%, 11,8 \%$ e $31,3 \%$, respectivamente. No entanto, o parasitismo por $A$. sagax em $S$. derogata não teve efeito sobre a
RGR e RMR são reduzidos em conseqüência do ínstar adicional. Entre lagartas parasitadas (Tabela 2), devido ao aumento no ganho de peso e na quantidade de alimento ingerido por lagartas de sete ínstares, o impacto do ínstar adicional nos índices nutricionais foi menor.

\section{Agradecimentos}

Ao Dr. Paul M. Marsh, Systematic Entomology Laboratory, USDA, pela identificação de Glyptapanteles muesebecki. 


\section{Literatura Citada}

Beckage, N.E. \& L.M. Riddiford. 1978. Developmental interactions between the tobacco hornworm Manduca sexta and its braconid parasite Apanteles congregatus. Entomol. Exp. Appl. 23: 139-151.

Beckage, N.E. \& T.J. Templeton. 1986. Physiological effects of parasitism by Apanteles congregatus in terminal-stage tobacco hornworm larvae. J. Insect Physiol. 32: 299-314.

Duodu, Y.A. \& F.F. Antoh. 1984. Effect of parasitism by Apanteles sagax on growth, food consumption and food utilization in Sylepta derogata larvae (Lepidoptera: Pyralidae). Entomophaga 29: 63-71.

Guillot, F.S. \& S.B. Vinson. 1973. Effect of parasitism by Cardiochiles nigriceps on food consumption and utilization by Heliothis virescens. J. Insect Physiol. 19: 2073-2082

Hill, M.G. 1986. Effects of Cotesia ruficrus (Braconidae : Hymenoptera) parasitism and rearing density on Mythimna separata (Noctuidae : Lepidoptera) food consumption, and implications for biological control. N.Z.J. Agric. Res. 29: 281-288.

Jalali, S.K., S.P. Singh \& C.R. Ballal. 1988. Effect of parasitism by Cotesia congregata on consumption and utilization of artificial diet by larvae of Spodoptera litura (Lepidoptera: Noctuidae). Indian Journal Agr. Sci. 58: 529-531.

Lazzari S.N. \& F.A. Lazzari. 1985. Ocorrência de Pseudaletia sequax Franclemont, 1951 (Lepidoptera: Noctuidae) e seus inimigos naturais em cevada (Hordeum sp.), no Paraná. An. Soc. Entomol. Brasil 14: 59-66.
Oliveira $\mathbf{F}^{\circ}$, J. 1984. Biologia do parasitóide Apanteles muesebecki Blanchard, 1947 (Hymenoptera: Braconidae) sobre lagartas de Pseudaletia sequax Franclemont, 1951 (Lepidoptera: Noctuidae). Tese Mestrado, Curitiba, Universidade Federal do Paraná, 72p.

Rahman, M. 1970. Effect of parasitism on food consumption of Pieris rapae larvae. J. Econ. Entomol. 63: 820-821.

Sato, Y., J. Tagawa \& T. Hidaka. 1986. Effects of the gregarious parasitoids Apanteles ruficrus and A. kariyai, on host growth and development. J. Insect Physiol. 32: 281-286.

Scriber, J.M. \& F. Slansky Jr. 1981. The nutritional ecology of immature insects. Annu. Rev. Entomol. 26: 183-211.

Senthamizhselvan, M. \& J. Muthukrishnan. 1989. Effect of parasitization by a gregarious and a solitary parasitoid on food consumption and utilization by Porthesia scintillans Walker (Lepidoptera: Lymantriidae) and Spodoptera exigua Hubner (Lepidoptera:Noctuidae). Parasitol. Res. 76: 166170.

Slansky Jr., F. 1978. Utilization of energy and nitrogen by larvae of the imported cabbageworm, Pieris rapae, as affected by parasitism by Apanteles glomeratus. Environ. Entomol. 7: 179-185.

Smith, C.L. \& Z. Smilowitz. 1976. Growth and development of Pieris rapae larvae parasitized by Apanteles glomeratus. Entomol. Exp. Appl. 19: 189-195.

Tanaka, T., S. Yagi \& Y. Nakamatu. 1992. Regulation of parasitoid sex allocation and host growth by Cotesia (Apanteles) kariyai (Hymenoptera:Braconidae). Ann. Entomol. Soc. Am. 85: 310-316.

Vinson, S.B. \& G.F. Iwantsch. 1980. Host 
regulation by insect parasitoids. Quart. Rev. Biol. 55: 143-165.

Waldbauer, G.P. 1968. The consumption and utilization of food by insects. Adv. Insect Physiol. 5: 229-288.

Recebido em 24/07/97. Aceito em 16/04/98. 